\title{
Distribution and significance of interstitial fibrosis and stroma-infiltrating B cells in tongue squamous cell carcinoma
}

\author{
XIAO-MEI LAO $^{1 *}$, YU-JIE LIANG ${ }^{1 *}$, YU-XIONG SU $^{2}$, SI-EN ZHANG $^{1}$, XI ZHOU $^{1}$ and GUI-QING LIAO ${ }^{1}$ \\ ${ }^{1}$ Department of Oral and Maxillofacial Surgery, Guanghua School of Stomatology, \\ Guangdong Provincial Key Laboratory of Stomatology, Sun Yat-sen University, Guangzhou, Guangdong 510055; \\ ${ }^{2}$ Division of Oral and Maxillofacial Surgery, Faculty of Dentistry, The University of Hong Kong, Hong Kong SAR, P.R. China
}

Received November 11, 2014; Accepted December 10, 2015

DOI: $10.3892 / \mathrm{ol} .2016 .4184$

\begin{abstract}
Inflammation and desmoplasia are frequently identified in the tumor microenvironment, and have been demonstrated to be effective modulators of malignant biological events. However, the mechanisms by which the inflammatory microenvironment and interstitial fibrosis interact with one another remain to be elucidated. The present study aimed to investigate the degree of inflammation and interstitial fibrosis in tongue squamous cell carcinoma (TSCC), and how this acts to affect the outcome of TSCC. Tissue samples from 93 cases of TSCC and paired tumor-adjacent non-neoplastic tongue epithelium, as well as 14 cases of epithelial dysplasia, were used. Interstitial collagen fibers were assessed using Masson's trichrome stain. Immunohistochemical identification of cancer-associated fibroblasts (CAFs) and stroma-infiltrating $B$ cells was performed via detection of $\alpha$-smooth muscle actin (SMA), vimentin, desmin and cluster of differentiation 19 (CD19). The clinicopathological significance and overall survival of the TSCC patients were statistically analyzed. Regularly distributed CAFs and $\mathrm{CD} 19^{+} \mathrm{B}$ cells were identified in the TSCC stroma, whereas no CAFs or CD19+ $\mathrm{B}$ cells were observed in epithelial dysplasia samples or paired tumor-adjacent non-neoplastic tongue epithelium samples. The distribution of interstitial collagen fibers and CAFs was closely associated with the tumor stage of the primary cancer, and high levels of $\mathrm{CD} 19^{+} \mathrm{B}$ cells together with low CAF infiltration were identified to be associated with favorable prognosis in TSCC. In conclusion, the inflammatory and interstitial fibrotic
\end{abstract}

Correspondence to: Professor Gui-Qing Liao, Department of Oral and Maxillofacial Surgery, Guanghua School of Stomatology, Guangdong Provincial Key Laboratory of Stomatology, Sun Yat-sen University, 56 Lingyuanxi Road, Guangzhou, Guangdong 510055, P.R. China

E-mail: drliaoguiqing@hotmail.com

*Contributed equally

Key words: cancer-associated fibroblasts, stroma-infiltrating B cells, interstitial fibrosis, inflammatory microenvironment, tongue squamous cell carcinoma microenvironments coexist in TSCC, and each has specific effects on disease outcome, individually or perhaps collectively. However, it remains to be determined exactly how the microenvironments affect one another in TSCC.

\section{Introduction}

As we learn more about cancer, it has become clear that, as well as cancer cells, tumors exhibit an additional dimension of complexity, known as the 'tumor microenvironment'. Typically, this consists of an extracellular matrix (ECM) scaffold that is populated by ostensibly normal cells, including fibroblast-like cells, endothelial cells and immune cells (1). The tumor microenvironment is morphologically defined by desmoplasia, angiogenesis, inflammation and immune response. Each of these has an integral role in the carcinogenic process (1). It is believed that $>90 \%$ of all cancers are caused by acquired somatic mutations and environmental factors, and germline mutation is the cause of carcinogenesis in $\sim 10 \%$ of cases (2).

A role for inflammation in carcinogenesis is now widely accepted (3). Lymphocytes are a significant component of mononuclear cell populations that infiltrate human malignant tumors. The majority of previous studies have focused on cytotoxic T cells, as these demonstrate the greatest antitumor potential. However, analyses of tumor-infiltrating B cells have identified the presence of these cells to be the next most important predictor of disease outcome (4-6).

Unresolved inflammation commonly results in chronic inflammatory diseases, including fibrosis, pancreatitis and inflammatory bowel syndrome, which are known to greatly increase the risk of cancer (3). A systemic sclerosis study revealed that $\mathrm{B}$ cells may have a significant role in fibrosis, based on the observation that ex vivo co-culture of blood $B$ cells and fibroblasts induced secretion of transforming growth factor $\beta 1$, interleukin (IL)-6, chemokine (C-C motif) ligand (CCL) 2 and collagen, as well as expression of $\alpha$-smooth muscle actin (SMA) and matrix metalloproteinase 9 in dermal fibroblasts (7). However, to the best of our knowledge, it remains unclear whether there is a similar role for B cells in cancer.

One particular cell type, carcinoma-associated fibroblasts (CAFs; alternatively known as activated fibroblasts, myofibroblasts or tumor-associated fibroblasts), has been prominently investigated, and have been identified to be the 
most abundant cells in the tumor microenvironment. They are typically recognized by their expression of $\alpha$-SMA, which is absent in normal dermal fibroblasts $(8,9)$. In solid tumors, including breast cancer, CAFs may account for up to $90 \%$ of the tumor mass and have been observed to correlate with a desmoplastic phenotype (10). Notably, within a tumor microenvironment infiltrated by immune cells, CAFs contribute to the maintenance of an inflammatory phenotype rather than being a passive bystander $(11,12)$. We speculated that, if tumor-infiltrating B cells functioned in interstitial fibrosis in malignancy, interaction with CAFs may be a potential mechanism through which this may occur.

The significance of the tumor microenvironment in TSCC is currently a topic of significant interest. CAFs and tumor-infiltrating B cells may affect the outcome of TSCC. to the best of our knowledge, the present study is the first to investigate the distribution and significance of interstitial fibrosis and stroma-infiltrating B cells in TSCC.

\section{Materials and methods}

Patients and tissue samples. The present study was conducted at the Department of Oral and Maxillofacial Surgery of the Hospital of Stomatology, Guangdong Provincial Key Laboratory of Stomatology, Sun Yat-sen University (Guangzhou, China). Prior written informed patient consent and approval from the Institutional Research Ethics Committee was obtained. The investigations were performed using paraffin-embedded TSCC samples and paired tumor-adjacent non-neoplastic tongue epithelium samples from 93 patients. In addition, 14 samples were obtained from 14 patients with epithelial dysplasia tongue mucosa

Image analysis of interstitial collagen fibers. Pathological evaluation of every case was performed following hematoxylin and eosin staining. Tumor-node-metastasis (TNM) staging was determined according to the 2002 International Union Against Cancer TNM classification of malignant tumors (13). Cancer cell differentiation was classified into one of three grades (well, moderate and poor) using the 2006 World Health Organization classification (14). Collagen fiber bundles were stained and distinguished from cell components using Masson's trichrome stain.

Quantification of interstitial collagen fibers was performed by digital image analysis using Adobe Photoshop version 7.0 (Adobe Systems, Inc., San Jose, CA, USA) and Image-Pro Plus version 6.0 (Media Cybernetics, Inc., Rockville, MD, USA). A total of five random areas stained with Masson's trichrome stain in each specimen (magnification, x200) were acquired (Fig. 1A), and the red epithelial components were excluded from the analysis area (Fig. 1B). Subsequently, the total area was cut from Fig. 1B, with the exception of the blue collagen fibers (Fig. 1C). The percentage collagen fiber content (\%CFC) in the tumor stroma was calculated using these last two images as follows: \%CFC $=$ (area of Fig. 1C/area of Fig. 1B) $\times 100$ The mean $\% \mathrm{CFC}$ of the five randomized selected areas was regarded as the CFC of the specimen.

Immunohistochemistry. Immunostaining was performed according to the manufacturer's protocols, using the following primary antibodies: Mouse monoclonal anti-human $\alpha$-SMA (catalog no., M0851; clone, 1A4; Dako, Glostrup, Denmark), rabbit monoclonal anti-human vimentin (catalog no., 5741; clone, D21H3; Cell Signaling Technology, Inc., Danvers, MA, USA), mouse monoclonal anti-human desmin (catalog no., M0760; clone, D33; Dako) and mouse monoclonal anti-human CD19 (catalog no., ab31947; clone, 2E2B6B10; Abcam, Cambridge, MA, USA) antibodies.

Briefly, the paraffin-embedded specimens were cut into $4-\mu \mathrm{m}$ sections and heated at $60^{\circ} \mathrm{C}$ for $2 \mathrm{~h}$, followed by dewaxing and rehydration. For heat-induced antigen retrieval (performed in a microwave oven at $240 \mathrm{~W}$ for $20 \mathrm{~min}$ ), $\alpha$-SMA and desmin were treated with $10 \mathrm{mM}$ Tris-1 ethylenediaminetetraacetic acid buffer ( $\mathrm{pH} 9$ ), and vimentin and CD19 were treated with $10 \mathrm{mM}$ citrate buffer $(\mathrm{pH} \mathrm{6)}$. The sections were subsequently treated with $3 \%$ hydrogen peroxide, followed by $5 \%$ bovine serum albumin. All slides were incubated with anti- $\alpha$-SMA (dilution, 1:100) and anti-desmin (dilution, 1:150) for $1 \mathrm{~h}$ at room temperature, and with anti-vimentin (dilution, 1:100) and anti-CD19 (dilution, 1:350) overnight at $4^{\circ} \mathrm{C}$. Immunoreactivity was detected using labeled polymer-horseradish peroxidase reagent conjugated to AffiniPure goat anti-mouse (catalog no., E035111-01) or AffiniPure goat anti-rabbit (catalog no., E030120-02) immunoglobulin (Ig)G secondary antibody (EarthOx Life Sciences, Millbrae, CA, USA) and visualized with diaminobenzidine. Negative control experiments were performed by replacing the primary antibody with phosphate-buffered saline.

Immunostaining of CAFs was assessed by evaluation of the staining intensity and percentage of $\alpha$-SMA. The percentage of immunopositive CAFs in non-inflammatory and non-endothelial stromal cells was recorded as follows: 0, no positive cells; $1,1-33 \%$ positive cells; $2,34-66 \%$ positive cells; and $3,67-100 \%$ positive cells. Staining intensity was considered to be 0 when there was no staining, 1 where positivity was observed only at magnification, $x 400,2$ in cases where staining was evident at $\times 200$ but not at $\times 100$ and 3 in fields where immunopositive cells were observed even at magnification, $\mathrm{x} 100$. Multiplication of the percentage and intensity scores composed the staining index of each specimen. This index was classified as negative (score 0), low (score 1-2), moderate (score 3-4) or high (score 6-9) $(15,16)$.

The lesion cores were initially scored by assessing the proportion of each core that comprised epithelia and stroma at low magnification, and subsequently scoring the number of positively stained $\mathrm{CD} 19^{+} \mathrm{B}$ cells within the core area (by direct counting up to 20 , or by estimation to the nearest 10 when $>20$ ) at magnification, $x 200$. Cores were scored as 0 (no cells present), 1 ( $1-5$ cells), 2 (6-19 cells) or 3 ( $\geq 20$ cells).

Statistical analysis. Fisher's least significant difference test and one factor analysis of variance were utilized for semi-quantitative image analysis of the collagen fibers in tissue samples. The association between the immune expression of each protein and clinical stage of disease, tumor (T) stage, lymph node metastasis, degree of cell differentiation and prognosis were evaluated using the Kruskal-Wallis test or Mann-Whitney U test. Multivariate survival analysis was performed with the Cox proportional hazards regression model. Survival curves were generated by the Kaplan-Meier 
Table I. Clinicopathological features of tongue squamous cell carcinoma patients and their association with $\alpha$-SMA and CD19 expression in the tumor microenvironment.

\begin{tabular}{|c|c|c|c|c|c|c|c|c|}
\hline \multirow[b]{2}{*}{ Characteristics } & \multicolumn{2}{|c|}{ Patients } & \multicolumn{2}{|c|}{ Collagen fiber content } & \multicolumn{2}{|c|}{$\alpha-\mathrm{SMA}$} & \multicolumn{2}{|c|}{ CD19 } \\
\hline & $\mathrm{n}$ & $\%$ & Mean $\pm \mathrm{SD}$ & P-value & Mean rank & P-value & Mean rank & $\mathrm{P}$-value \\
\hline Age, years & & & & 0.334 & & 0.813 & & 0.609 \\
\hline$<50$ & 42 & 45.2 & $0.10 \pm 0.09$ & & 47.70 & & 44.51 & \\
\hline$\geq 50$ & 51 & 54.8 & $0.12 \pm 0.10$ & & 46.42 & & 47.22 & \\
\hline Gender & & & & 0.215 & & 0.047 & & 0.687 \\
\hline Male & 56 & 60.2 & $0.13 \pm 0.11$ & & 51.35 & & 45.12 & \\
\hline Female & 37 & 39.8 & $0.10 \pm 0.09$ & & 40.42 & & 47.28 & \\
\hline Clinical stage & & & & 0.239 & & 0.374 & & 0.912 \\
\hline I-II & 51 & 54.8 & $0.10 \pm 0.09$ & & 43.44 & & 44.25 & \\
\hline III-IV & 42 & 45.2 & $0.13 \pm 0.10$ & & 48.19 & & 44.83 & \\
\hline Tumor stage & & & & 0.031 & & 0.001 & & 0.479 \\
\hline $1-2$ & 78 & 85.7 & $0.10 \pm 0.10$ & & 42.34 & & 44.27 & \\
\hline $3-4$ & 13 & 14.3 & $0.17 \pm 0.09$ & & 67.69 & & 49.69 & \\
\hline Node stage & & & & 0.966 & & 0.209 & & 0.207 \\
\hline 0 & 63 & 69.2 & $0.11 \pm 0.11$ & & 48.23 & & 47.22 & \\
\hline $1-3$ & 28 & 30.8 & $0.11 \pm 0.10$ & & 40.98 & & 40.16 & \\
\hline Pathological differentiation & & & & 0.231 & & 0.125 & & 0.217 \\
\hline Well-differentiated & 47 & 50.5 & $0.10 \pm 0.09$ & & 39.80 & & 44.64 & \\
\hline Moderately differentiated & 40 & 43.0 & $0.14 \pm 0.11$ & & 52.84 & & 46.56 & \\
\hline Poorly differentiated & 6 & 6.5 & $0.10 \pm 0.10$ & & 44.00 & & 26.50 & \\
\hline Overall survival & & & & 0.865 & & 0.361 & & 0.011 \\
\hline Alive & 39 & 41.9 & $0.12 \pm 0.11$ & & 30.86 & & 35.95 & \\
\hline Succumbed to disease & 25 & 26.9 & $0.11 \pm 0.09$ & & 35.06 & & 24.46 & \\
\hline Missing & 29 & 31.2 & $0.11 \pm 0.11$ & & & & & \\
\hline
\end{tabular}

Collagen fiber content is expressed as a ratio of the percentage of collagen fiber content in the tumor stroma. SMA, smooth muscle actin; CD, cluster of differentiation; SD, standard deviation; Mean rank, the mean as determined by the rank sum test.
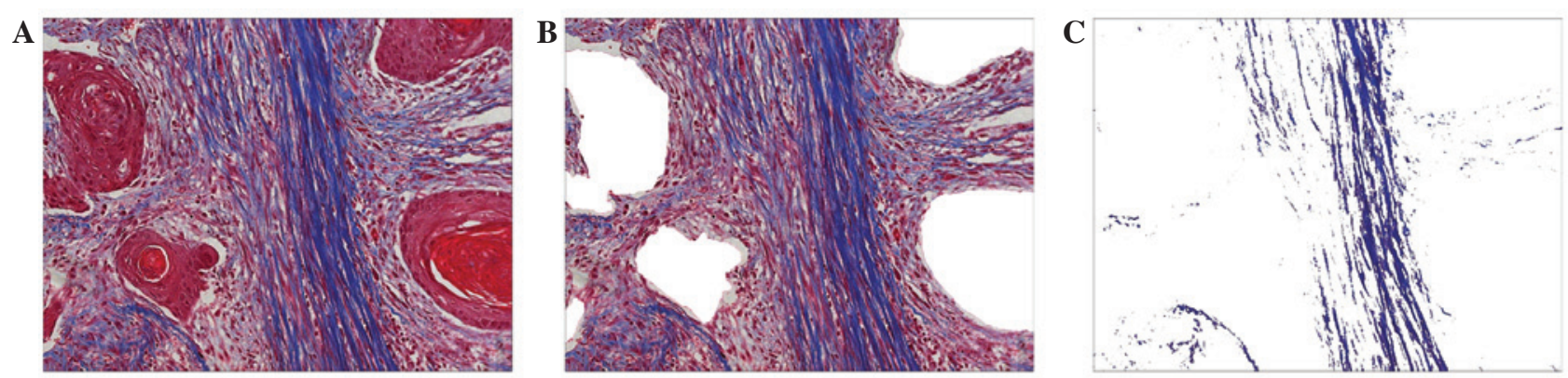

Figure 1. Semi-quantification image analysis of stromal collagen fibers. (A) Original Masson's trichrome stained image of the tissue section. Distinction of the cell compartment (red) and collagen fiber (blue) is clear. (B) Area of the stroma from image A demonstrating removal of the epithelial cell compartments. (C) Area of the interstitial collagen fiber compartment; all other areas removed, with the exception of the fibrous area from image B. Magnification, $\mathrm{x} 200$.

method and compared using the log-rank test. $\mathrm{P}<0.05$ was considered to indicate a statistically significant difference.

\section{Results}

Summary of patients. A total of 107 samples (93 TSCC and 14 epithelial dysplasia) from patients aged 20-80 years (mean age, 52 years) were evaluated in the present study. The median follow-up time of the TSCC patients was 41 months. The clinical profiles of the TSCC patients included in the present study are summarized in Table I.

Image analysis of interstitial collagen fibers indicates an association between CFC and certain clinicopathological 

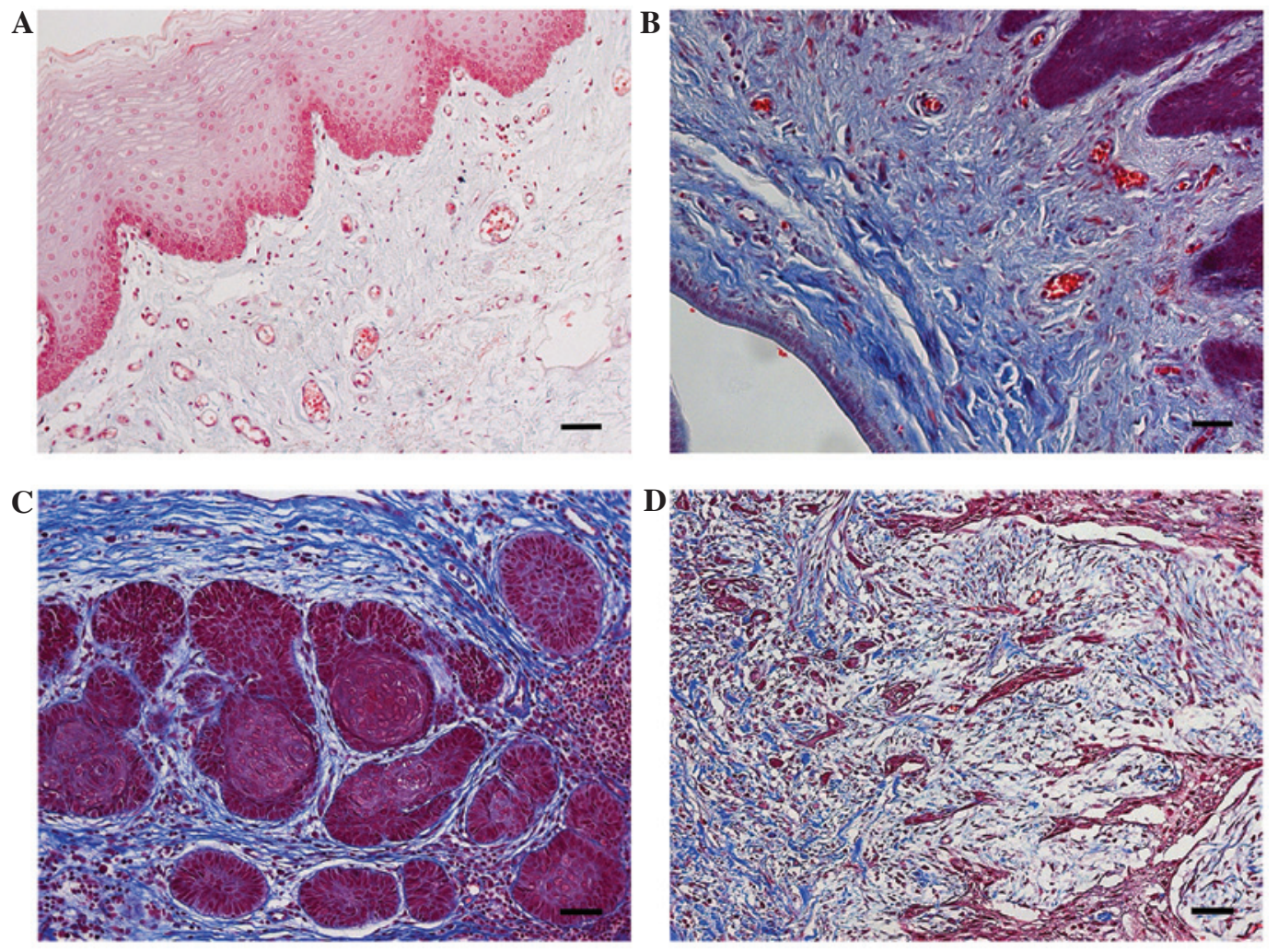

Figure 2. Distribution patterns of interstitial collagen fibers in the tissue samples. (A) No positive staining of interstitial collagen fibers was observed in the paired tumor-adjacent non-neoplastic tongue epithelium samples. (B) In the epithelial dysplasia samples, profuse interstitial collagen fibers were identified. (C) Dense and well-organized, parallel bundles of collagen fibers surrounding the tumor nest. (D) Complex fibers existed in large quantities around the cancer cells that were in the stroma. Magnification, x200.

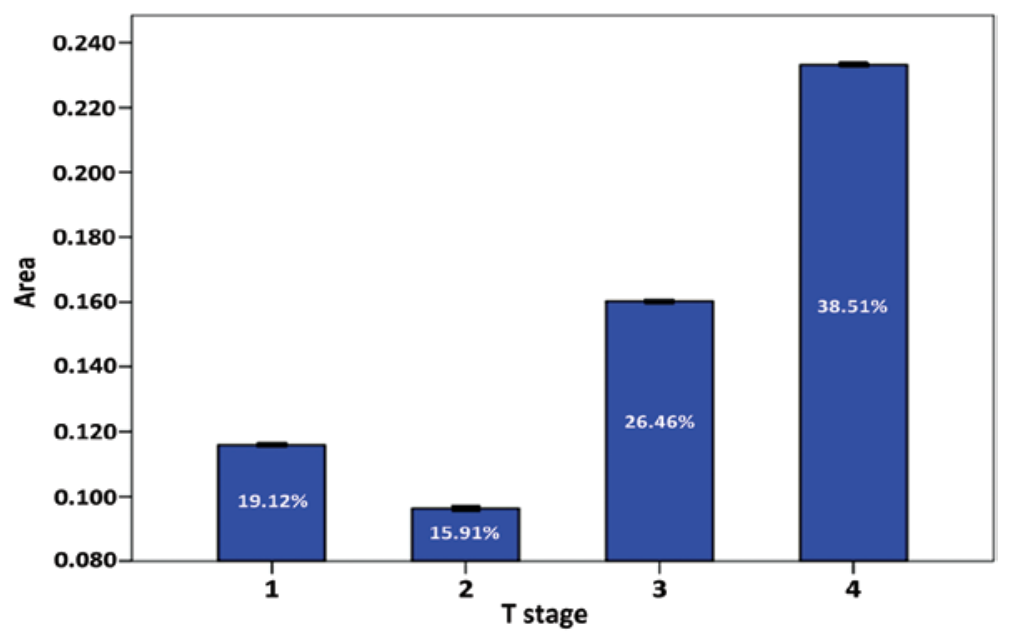

Figure 3. Association between average \% CFC and the T stage of tongue squamous cell carcinoma. Tumor interstitial CFC indicated a trend for increasing as the tumor increased in size, with the exception of $\mathrm{T} 1$ stage cases. The data is presented as the mean \pm standard deviation. CFC, collagen fiber content; T, tumor.

features. The blue stained areas in the tissue sections following Masson's trichrome staining represented interstitial collagen fibers. No positive staining was detected in the paired tumor-adjacent non-neoplastic samples (Fig. 2A); however, in the epithelial dysplasia samples, a large number of interstitial collagen fibers were observed (Fig. 2B). A broad array of collagen fiber architectures were identified in TSCC samples, with certain samples exhibiting well-organized bundles surrounding epithelial clusters in the tissue section (Fig. 2C), and others in which interstitial collagen fibers were observed to intercalate between individual epithelial cells and interact with regions displaying poor tumor margins (Fig. 2D).

Analysis of CFC vs. clinicopathological criteria indicated a significant association between the T stage of TSCC and $\mathrm{CFC}(\mathrm{P}=0.031$; Fig. 3). The degree of CFC did not affect the overall survival rate of patients included in the present study (Table I).

Distribution patterns of CAFs vary with tissue sample type. Stromal spindle cells positive for $\alpha$-SMA and vimentin, 
Table II. Immunohistochemical staining index for $\alpha$-SMA and CD19 in ED, TSCC and PTANNE.

\begin{tabular}{|c|c|c|c|c|c|c|c|c|c|c|c|c|}
\hline \multirow{2}{*}{$\begin{array}{l}\text { Tissue } \\
\text { type }\end{array}$} & \multicolumn{4}{|c|}{$\alpha$-SMA, n } & \multirow[b]{2}{*}{ P-value } & \multirow[b]{2}{*}{$\mathrm{R}$-value } & \multicolumn{4}{|c|}{$\mathrm{CD} 19, \mathrm{n}$} & \multirow[b]{2}{*}{ P-value } & \multirow[b]{2}{*}{$\mathrm{R}$-value } \\
\hline & Negative & Low & Moderate & High & & & 0 & 1 & 2 & 3 & & \\
\hline ED & 14 & 0 & 0 & 0 & & & 14 & 0 & 0 & 0 & & \\
\hline PTANNE & 93 & 0 & 0 & 0 & $<0.001$ & -0.720 & 93 & 0 & 0 & 0 & $<0.001$ & -0.872 \\
\hline TSCC & 16 & 30 & 26 & 21 & & & 7 & 29 & 34 & 23 & & \\
\hline
\end{tabular}

P-values were calculated using Spearman's rank correlation analysis. SMA, smooth muscle actin; CD, cluster of differentiation; ED, epithelial dysplasia; PTANNE, paired tumor-adjacent non-neoplastic epithelium; TSCC, tongue squamous cell carcinoma.

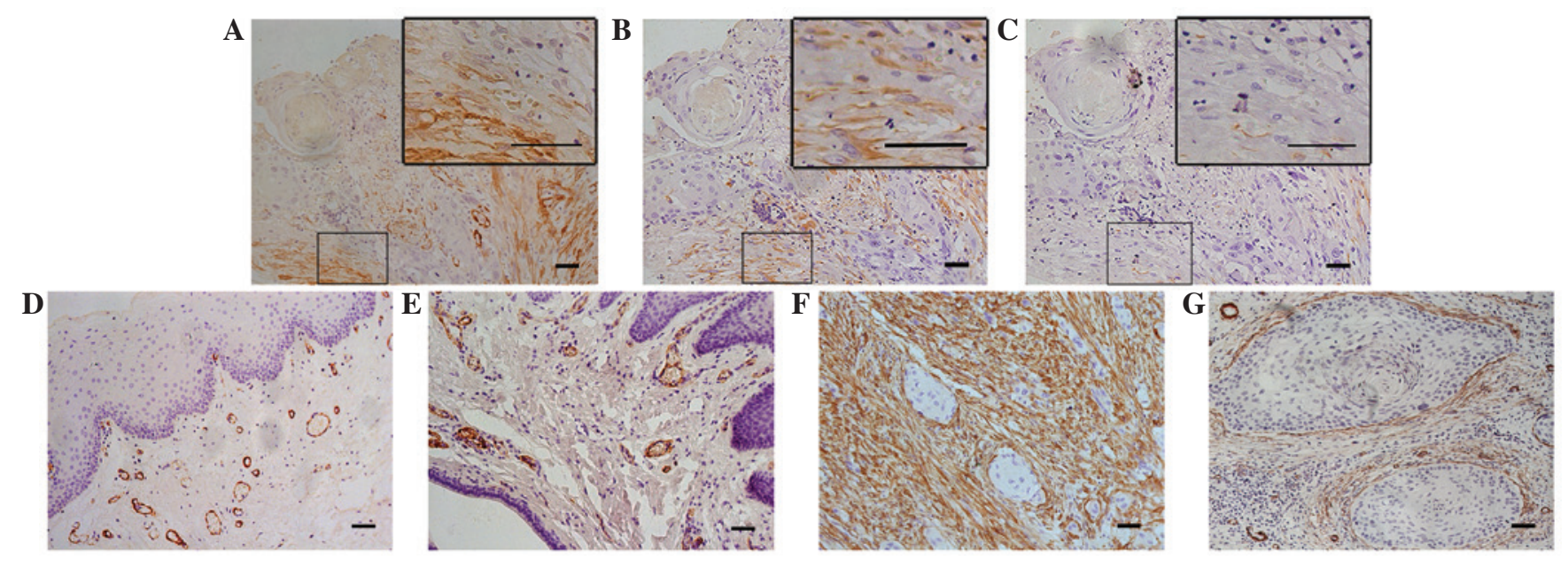

Figure 4. Immunohistochemical staining of CAFs. CAFs were (A) $\alpha$-SMA and (B) vimentin positive, but (C) desmin negative. (D and E) Negative staining of $\alpha$-SMA in the epithelial dysplasia and paired tumor-adjacent non-neoplastic tongue epithelium samples. (F) Typical 'network' pattern formed when CAFs are particularly abundant and occupy almost the entire tumor stroma. (G) 'Spindle' pattern, in which CAFs are arranged in 1-3 rows in a regular order in the periphery of the neoplastic islands. CAFs, cancer-associated fibroblasts; SMA, smooth muscle actin.

but negative for desmin, were regarded as CAFs (Fig. 4). One consistent feature of CAFs in desmoplastic lesions is a myofibroblast phenotype, which is based on the synthesis of intracellular smooth muscle-like proteins, in particular $\alpha$-SMA (17). Therefore, the present study assessed CAFs by evaluating the staining intensity and percentage of $\alpha$-SMA.

For immunostaining of $\alpha$-SMA, blood vessels present within the connective tissue of the immunostained sections served as a positive internal control and stained with an intensity of 3 in all cases.

Despite the distribution of profuse interstitial collagen fibers, no positive staining of $\alpha$-SMA was detected in the epithelial dysplasia samples or the paired tumor-adjacent non-neoplastic samples, with the exception of blood vessels (Fig. 4D and E). Thus, CAFs were not considered to be present in these tissue sample types. In TSCC samples, in non-inflammatory and non-endothelial stromal cells immediately adjacent to carcinomatous islands, the $\alpha$-SMA staining index was negative in $17.2 \%$, low in $32.3 \%$, moderate in $28.0 \%$ and high in $22.6 \%$ of all cases investigated. The distribution of CAFs and interstitial collagen fibers was identified to be highly homogeneous between \%CFC and $\alpha$-SMA staining index $(\mathrm{P}<0.001$; Table II), and a trend for increasing amounts of $\alpha$-SMA-positive CAFs was clearly demonstrated with regard to the increasing $\mathrm{T}$ stage of TSCC patients $(\mathrm{P}=0.001)$, which was consistent with results achieved with Masson's trichrome staining.

Additional investigation of CAF distribution in different samples revealed a highly heterogeneous pattern, even within a single tumor, and 'spindle' and 'network' patterns were observed. In the 'network' pattern, CAFs were particularly abundant and occupied almost the entire tumor stroma (Fig. 4F). The 'spindle' pattern was characterized by stromal CAFs that were located at the periphery of carcinomas in 1-3 concentric layers; this pattern was additionally observed to be adjacent to a number of tumor islands/nests (Fig. 4G). However, no significant difference between the two distribution patterns with regard to the survival benefit of the disease was identified in the present study $(\mathrm{P}=0.560)$.

Distribution patterns of $\mathrm{CD} 9^{+}$stroma-infiltrating $\mathrm{B}$ cells vary with tissue sample type. $\mathrm{CD} 19^{+}$stroma-infiltrating B cells were identified only in TSCC samples, even in tumors exhibiting low densities of the cellular components of the ECM. $\mathrm{CD} 9^{+}$stroma-infiltrating B cells were primarily enriched in the invasive margin; however, only small fractions of B cells sporadically infiltrated into the peritumoral areas. The majority of $\mathrm{CD}_{19}{ }^{+}$stroma-infiltrating $\mathrm{B}$ cells resided in follicular aggregates, resembling the previously reported 'Crohn's-like reaction' model of tumor-infiltrating lymphocytes (TILs; Fig. 5) (18). 

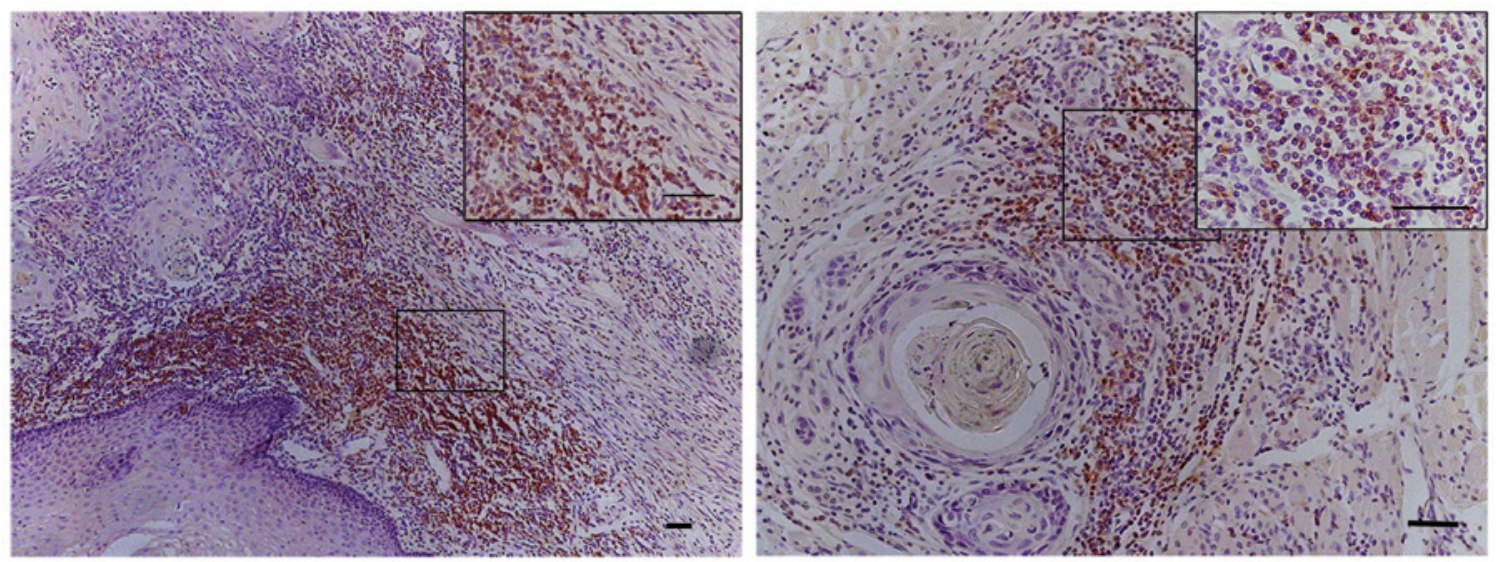

Figure 5. Follicular aggregates of stroma-infiltrating B cells in the stroma of tongue squamous cell carcinoma, exhibiting the 'Crohn's-like reaction'.

A

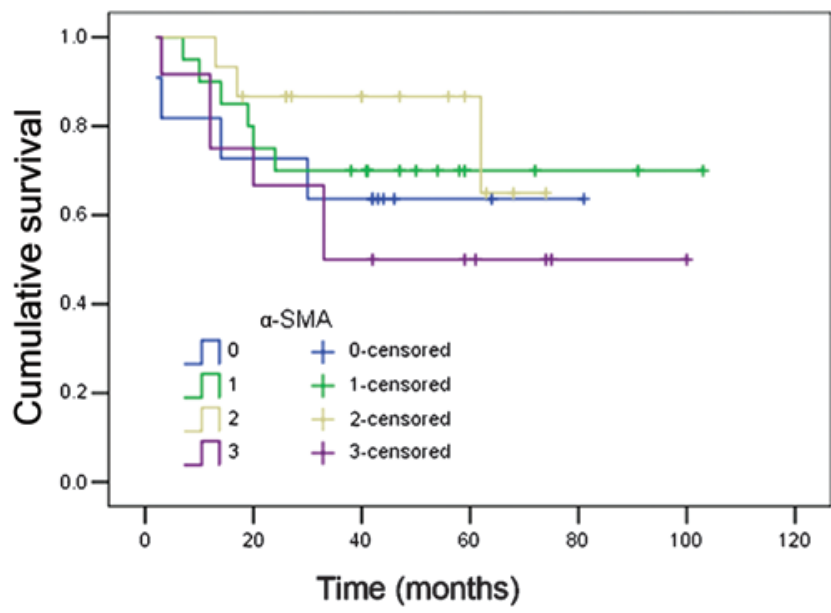

B

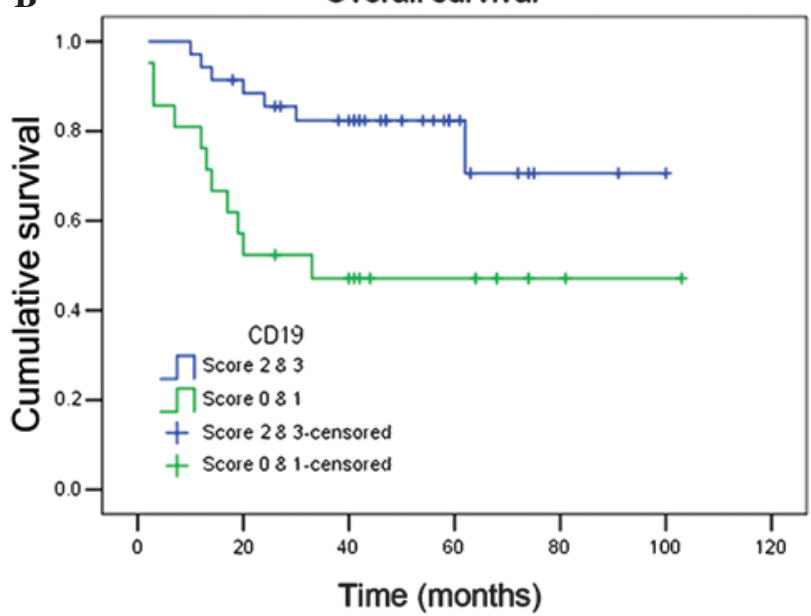

Figure 6. Kaplan-Meier survival curves for tongue squamous cell carcinoma patients according to (A) $\alpha$-SMA and (B) CD19 staining in the tumor stroma. The $\log$-rank test revealed that the survival rate between various scores of $\alpha$-SMA staining was not statistically significant $(\mathrm{P}=0.504)$, however, a statistically significant difference was observed between various scores of $\mathrm{CD} 19$ expression $(\mathrm{P}=0.008)$. SMA, smooth muscle actin; $\mathrm{CD}$, cluster of differentiation.

Statistical analysis of $\mathrm{CD} 19^{+}$stroma-infiltrating B cell density revealed a significant difference between TSCC samples and epithelial dysplasia samples or paired tumor-adjacent non-neoplastic samples. A positive correlation between the density of $\mathrm{CD} 19^{+}$stroma-infiltrating B cells and overall survival of TSCC patients was identified $(\mathrm{P}=0.008$; Fig. 6).

Chronic inflammatory fibrotic microenvironments of TSCC may be associated with patient survival. CAFs and CD19 ${ }^{+}$ stroma-infiltrating B cells were observed to coexist in the TSCC stroma, however, they did not in the paired tumor-adjacent non-neoplastic samples nor the epithelial dysplasia samples. In a number of cases, there was a tumor capsule-like structure formed by fibrous connective tissue surrounding the neoplastic mass, and an uneven distribution of $\mathrm{CD} 19^{+}$stroma-infiltrating $\mathrm{B}$ cells was observed. In certain cases, $\mathrm{CD} 19^{+}$stroma-infiltrating B cells only assembled outside the tumor capsule, whereas in other cases they were identified on both sides. In these cases, $\mathrm{CD} 19^{+}$stroma-infiltrating B cells appeared to have penetrated the tumor capsule and were able to interact directly with tumor cells. Statistical analysis revealed that TSCC cases exhibiting severe desmoplasia and low inflammatory status possessed the lowest overall survival rate, and cases demonstrating low desmoplasia and a high inflammatory degree had significantly improved overall survival rates in comparison $(\mathrm{P}=0.040)$.

\section{Discussion}

To the best of our knowledge, the present study is the first to investigate the distribution of interstitial fibrosis and stroma-infiltrating B cells in the stroma of epithelial dysplasia, TSCC and paired tumor-adjacent non-neoplastic samples, and to analyze their clinical significance. The coexistence of two significant features in the TSCC microenvironment was investigated, and it was identified that CAFs and CD19 ${ }^{+}$ stroma-infiltrating B cells were associated with the clinicopathological characteristics and overall survival of TSCC patients.

In the present study, massively disseminated CAFs were observed in the TSCC stroma, whereas no CAFs were detected in the epithelial dysplasia and paired tumor-adjacent non-neoplastic epithelium samples. The presence of CAFs was 
observed to be positively correlated with the T stage of these patients, indicating a tumor-promoting function of CAFs. This result was consistent with the results of previous studies, which had observed that CAFs had a tumor-promoting role and protumorigenic properties $(19,20)$. Fibrotic histological alterations of the stroma may range from a predominantly cellular stroma with little ECM, to a dense tissue with a minimum of cells and a maximum content of matrix (21). In breast cancer, high fibrillar collagen density correlates with increased mammographic density in women, which augments breast cancer risk four-fold (22).

Highly heterogeneous arrangements of CAFs and collagen fibers were observed to be present in the stroma of TSCCs. Even with identical degrees of CAFs/interstitial fibrosis, the organization of these components may vary significantly. Well-formed 'spindle' or 'network' patterns were observed, as well as disorganised and chaotic patterns. However, statistical analysis revealed no significant difference between the various patterns with regard to the 3-year survival rate of TSCC patients. Agarwal et al (23) identified that, as well as the arrangement, the type of collagen fiber was important. In an immunohistochemical study involving 35 tissue blocks, a direct association between the presence of type IV collagen and the differentiation degree of SCC cells was confirmed (23). Additional investigation concerning various distribution patterns of CAFs or specific types of interstitial collagen fibers in the TSCC microenvironment is required.

An additional notable finding of the present study was the identification of a positive correlation between the density of $\mathrm{CD} 9^{+}$stroma-infiltrating B cells and overall survival. Density of $\mathrm{CD}_{19}{ }^{+}$stroma-infiltrating B cells was an independent prognostic factor among all clinicopathological features studied. The impact of stroma-infiltrating B cells in carcinogenesis has begun to be investigated. In particular, two specific subtypes of stroma-infiltrating B cells, tumor-evoked regulatory B cells (tBregs) and CD20+ TIL-B cells have attracted interest. There is no unique marker, or set of markers, that exclusively identifies tBregs, and they are defined as $\mathrm{CD} 19^{+} \mathrm{B}$ cells that are $\mathrm{CD} 25^{\mathrm{High}}$, B7-H11 ${ }^{\mathrm{High}}, \mathrm{CD} 81^{\mathrm{High}}, \mathrm{CD} 86^{\mathrm{High}}, \mathrm{CCL} 6^{\mathrm{High}}, \mathrm{CD} 62 \mathrm{~L}^{\text {Low }}$ and $\operatorname{Ig} \mathrm{M}^{\text {Int/Low }}(24)$. It has been proposed that tBregs demonstrate tumor-promoting action during squamous carcinogenesis (25). However, the present study revealed that $\mathrm{CD} 19^{+} \mathrm{B}$ cells were a favorable prognostic factor, which is similar to $\mathrm{CD} 20^{+} \mathrm{B}$ cells. Previous studies demonstrated that $\mathrm{CD} 20^{+} \mathrm{B}$ cells possessed a tumor suppressing function in breast and ovarian cancer, as well as hepatocellular carcinoma $(5,6,26,27)$. The discrepancy may be explained by the hypothesis that $\mathrm{CD} 19^{+} \mathrm{B}$ cells have varying roles between different tumor cell types or tumor stages. This additionally indicates that the exact function of stroma-infiltrating B cells in carcinogenesis and cancer progression is more complicated than expected, and additional studies are required to reveal the underlying mechanism.

The results of the present study demonstrated highly heterogeneous distribution patterns of interstitial collagen fibers and stroma-infiltrating B cells in TSCC, and the appearance of the two was not necessarily isochronous. The distribution pattern of a large amount of interstitial fibrosis and low levels of $\mathrm{CD} 19^{+} \mathrm{B}$ cell infiltration resulted in reduced overall survival. This notable finding may indicate the occurrence of cross-talk between CAFs and $\mathrm{CD} 19^{+} \mathrm{B}$ cells. It is believed that CAFs are initially educated by immune cells, due to the release of IL-1 $\beta$, during the early stages of tumorigenesis, and as tumor progression occurs, they acquire the ability to recruit and regulate immune cells to an eventual immune-suppressed phenotype that is compatible with disease progression $(28,29)$.

There are certain limitations in the present study, and the association between inflammation and interstitial fibrosis in the tumor microenvironment is complicated. The present study provided only preliminary results that indicated a coexistence of inflammation and interstitial fibrosis in the TSCC microenvironment. Additional studies are required to investigate the presence of $\mathrm{CD} 19^{+} \mathrm{B}$ cells, as well as the presence of $\mathrm{CD} 20^{+} \mathrm{B}$ cells, tBregs and other cells in TSCC. Furthermore, additional studies are required in vivo to elucidate the dynamic interactions and exact roles of these cells in TSCC.

In conclusion, the present study revealed that the degree of CAFs/interstitial collagen fibers correlates with the $\mathrm{T}$ stage of TSCC, and the existence of $\mathrm{CD} 9^{+}$stroma-infiltrating $B$ cells may serve a functional role in the TSCC inflammatory microenvironment. Distribution patterns of interstitial fibrosis and $\mathrm{CD} 19^{+} \mathrm{B}$ cells are associated with overall survival of TSCC patients. A pattern of high interstitial fibrosis and low levels of $\mathrm{CD} 19^{+} \mathrm{B}$ cells correlated with the lowest overall survival rate. The results of the present study may assist with additional investigation of inflammation and interstitial fibrosis in the TSCC microenvironment.

\section{Acknowledgements}

The authors would like to thank Mr. Tao He (Guangdong Provincial Key Laboratory of Stomatology, Sun Yat-sen University) for technical assistance. The present study was supported by grants from the National Natural Science Foundation of China (grant nos. 81172566, 81372884 and 81302367). Abstract previously published in the proceedings of the 5th World Congress of the International Academy of Oral Oncology in São Paulo, 2015.

\section{References}

1. Albini A and Sporn MB: The tumour microenvironment as a target for chemoprevention. Nat Rev Cancer 7: 139-147, 2007.

2. Jemal A, Siegel R, Xu J and Ward E: Cancer statistics, 2010. CA Cancer J Clin 60: 277-300, 2010.

3. Aggarwal BB, Vijayalekshmi RV and Sung B: Targeting inflammatory pathways for prevention and therapy of cancer: Short-term friend, long-term foe. Clin Cancer Res 15: 425-430, 2009.

4. Erdag G, Schaefer JT, Smolkin ME, Deacon DH, Shea SM, Dengel LT, Patterson JW and Slingluff CL Jr: Immunotype and immunohistologic characteristics of tumor-infiltrating immune cells are associated with clinical outcome in metastatic melanoma. Cancer Res 72: 1070-1080, 2012.

5. Nielsen JS, Sahota RA, Milne K, Kost SE, Nesslinger NJ, Watson $\mathrm{PH}$ and Nelson $\mathrm{BH}$ : CD20+ tumor-infiltrating lymphocytes have an atypical CD27-memory phenotype and together with $\mathrm{CD} 8+\mathrm{T}$ cells promote favorable prognosis in ovarian cancer. Clin Cancer Res 18: 3281-3292, 2012.

6. Shi JY, Gao Q, Wang ZC, Zhou J, Wang XY, Min ZH, Shi YH, Shi GM, Ding ZB, Ke AW, et al: Margin-infiltrating CD20(+) $B$ cells display an atypical memory phenotype and correlate with favorable prognosis in hepatocellular carcinoma. Clin Cancer Res 19: 5994-6005, 2013.

7. François A, Chatelus E, Wachsmann D, Sibilia J, Bahram S, Alsaleh G and Gottenberg JE: B lymphocytes and B-cell activating factor promote collagen and profibrotic markers expression by dermal fibroblasts in systemic sclerosis. Arthritis Res Ther 15: R168, 2013. 
8. Shimoda M, Mellody KT and Orimo A: Carcinoma-associated fibroblasts are a rate-limiting determinant for tumour progression. Semin Cell Dev Biol 21: 19-25, 2010.

9. Räsänen K and Vaheri A: Activation of fibroblasts in cancer stroma. Exp Cell Res 316: 2713-2722, 2010.

10. Tripathi M, Billet S and Bhowmick NA: Understanding the role of stromal fibroblasts in cancer progression. Cell Adh Migr 6: 231-235, 2012.

11. DeFilippis RA, Chang H, Dumont N, Rabban JT, Chen YY, Fontenay GV, Berman HK, Gauthier ML, Zhao J, Hu D, et al: CD36 repression activates a multicellular stromal program shared by high mammographic density and tumor tissues. Cancer Discov 2: 826-839, 2012.

12. Wheeler SE, Shi H, Lin F, Dasari S, Bednash J, Thorne S Watkins S, Joshi R and Thomas SM: Enhancement of head and neck squamous cell carcinoma proliferation, invasion, and metastasis by tumor-associated fibroblasts in preclinical models. Head Neck 36: 385-392, 2014.

13. Sobin LH and Wittekind C (eds): TNM Classification of Malignant Tumours. 6th edition. John Wiley \& Sons, Hoboken, NJ, 2002.

14. Thompson L: World Health Organization classification of tumours: Pathology and genetics of head and neck tumours. Ear Nose Throat J 85: 74, 2006

15. Etemad-Moghadam S, Khalili M, Tirgary F and Alaeddini M: Evaluation of myofibroblasts in oral epithelial dysplasia and squamous cell carcinoma. J Oral Pathol Med 38: 639-643, 2009.

16. Chaudhary M, Gadbail AR, Vidhale G, Mankar Gadbail MP, Gondivkar SM, Gawande M and Patil S: Comparison of myofibroblasts expression in oral squamous cell carcinoma, verrucous carcinoma, high risk epithelial dysplasia, low risk epithelial dysplasia and normal oral mucosa. Head Neck Pathol 6: 305-313, 2012.

17. Thode C, Jørgensen TG, Dabelsteen E, Mackenzie I and Dabelsteen S: Significance of myofibroblasts in oral squamous cell carcinoma. J Oral Pathol Med. 40: 201-207, 2011.

18. Maletzki C, Jahnke A, Ostwald C, Klar E, Prall F and Linnebacher M: Ex-vivo clonally expanded B lymphocytes infiltrating colorectal carcinoma are of mature immunophenotype and produce functional IgG. PLoS One. 7: e32639, 2012.
19. Dumont N, Liu B, Defilippis RA, Chang H, Rabban JT, Karnezis AN, Tjoe JA, Marx J, Parvin B and Tlsty TD: Breast fibroblasts modulate early dissemination, tumorigenesis and metastasis through alteration of extracellular matrix characteristics. Neoplasia 15: 249-262, 2013.

20. De Wever O, Van Bockstal M, Mareel M, Hendrix A and Bracke M: Carcinoma-associated fibroblasts provide operational flexibility in metastasis. Semin Cancer Biol 25: 33-46, 2014.

21. Tlsty TD and Coussens LM: Tumor stroma and regulation of cancer development. Annu Rev Pathol 1: 119-150, 2006.

22. Li T, Sun L, Miller N, Woo J, Hulse-Smith L, Tsao MS, Khokha R, Martin L and Boyd N: The association of measured breast tissue characteristics with mammographic density and other risk factors for breast cancer. Cancer Epidemiol Biomarkers Prev 14: 343-349, 2005

23. Agarwal P and Ballabh R: Expression of type IV collagen in different histological grades of oral squamous cell carcinoma: An immunohistochemical study. J Cancer Res Ther 9: 272-275, 2013.

24. Olkhanud PB, Damdinsuren B, Bodogai M, Gress RE, Sen R, Wejksza K, Malchinkhuu E, Wersto RP and Biragyn A: Tumor-evoked regulatory $\mathrm{B}$ cells promote breast cancer metastasis by converting resting $\mathrm{CD} 4^{+} \mathrm{T}$ cells to T-regulatory cells. Cancer Res 71: 3505-3515, 2011.

25. Fremd C, Schuetz F, Sohn C, Beckhove P and Domschke C: $\mathrm{B}$ cell-regulated immune responses in tumor models and cancer patients. Oncoimmunology 2: e25443, 2013.

26. Nelson BH: CD20+ B cells: The other tumor-infiltrating lymphocytes. J Immunol 185: 4977-4982, 2010.

27. Mahmoud SM, Lee AH, Paish EC, Macmillan RD, Ellis IO and Green AR: The prognostic significance of B lymphocytes in invasive carcinoma of the breast. Breast Cancer Res Treat 132: 545-553, 2012.

28. Erez N, Truitt M, Olson P, Arron ST and Hanahan D: Cancer-associated fibroblasts are activated in incipient neoplasia to orchestrate tumor-promotinginflammation in an NF-kappaB-dependent manner. Cancer Cell 17: 135-147, 2010.

29. De Wever O, Demetter P, Mareel M and Bracke M: Stromal myofibroblasts are drivers of invasive cancer growth. Int J Cancer 123: 2229-2238, 2008. 